\title{
Patient-Reported Outcomes and Preferences for Colon Capsule Endoscopy and Colonoscopy: A Systematic Review with Meta-Analysis
}

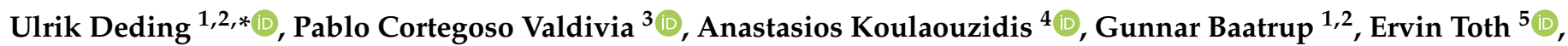 \\ Cristiano Spada ${ }^{6}$, Ignacio Fernández-Urién ${ }^{7}$, Marco Pennazio ${ }^{8}\left(\mathbb{D}\right.$ and Thomas Bjørsum-Meyer ${ }^{1,2}$
}

1 Department of Clinical Research, University of Southern Denmark, 5230 Odense, Denmark; gunnar.baatrup@rsyd.dk (G.B.); thomas.bjoersum-meyer@rsyd.dk (T.B.-M.)

2 Department of Surgery, Odense University Hospital, 5000 Odense, Denmark

3 Gastroenterology and Endoscopy Unit, University Hospital of Parma, University of Parma, 43121 Parma, Italy; cortegosopablo@yahoo.it

4 Department of Social Medicine \& Public Health, Faculty of Health Sciences, Pomeranian Medical University, 70-204 Szczecin, Poland; akoulaouzidis@hotmail.com

5 Department of Gastroenterology, Skåne University Hospital, Lund University, 22100 Malmö, Sweden; ervin.toth@med.lu.se

6 Digestive Endoscopy Unit, Fondazione Poliambulanza Istituto Ospedaliero, 25133 Brescia, Italy; cristianospada@gmail.com

check for

updates

Citation: Deding, U.; Cortegoso Valdivia, P.; Koulaouzidis, A.; Baatrup, G.; Toth, E.; Spada, C.; Fernández-Urién, I.; Pennazio, M.; Bjørsum-Meyer, T. Patient-Reported Outcomes and Preferences for Colon Capsule Endoscopy and Colonoscopy: A Systematic Review with Meta-Analysis. Diagnostics 2021, 11, 1730. https://doi.org/10.3390/ diagnostics11091730

Academic Editor: Yunjeong Lim

Received: 20 August 2021

Accepted: 18 September 2021

Published: 20 September 2021

Publisher's Note: MDPI stays neutral with regard to jurisdictional claims in published maps and institutional affiliations.

Copyright: (c) 2021 by the authors. Licensee MDPI, Basel, Switzerland. This article is an open access article distributed under the terms and conditions of the Creative Commons Attribution (CC BY) license (https:// creativecommons.org/licenses/by/ $4.0 /)$.
7 Department of Gastroenterology, Complejo Hospitalario de Navarra, 31008 Pamplona, Spain; ifurien@yahoo.se

8 University Division of Gastroenterology, AOU Città Della Salute e Della Scienza, University of Turin, 10124 Turin, Italy; pennazio.marco@gmail.com

* Correspondence: ulrik.deding@rsyd.dk; Tel.: +45-4257-4243

\begin{abstract}
Colon capsule endoscopy as an alternative to colonoscopy for the diagnosis of colonic disease may serve as a less invasive and more tolerable investigation for patients. Our aim was to examine patient-reported outcomes for colon capsule endoscopy compared to conventional optical colonoscopy including preference of investigation modality, tolerability and adverse events. A systematic literature search was conducted in Web of Science, PubMed and Embase. Search results were thoroughly screened for in- and exclusion criteria. Included studies underwent assessment of transparency and completeness, after which, data for meta-analysis were extracted. Pooled estimates of patient preference were calculated and heterogeneity was examined including univariate meta-regressions. Patient-reported tolerability and adverse events were reviewed. Out of fourteen included studies, twelve had investigated patient-reported outcomes in patients who had undergone both investigations, whereas in two the patients were randomized between investigations. Pooled patient preferences were estimated to be $52 \%$ (CI 95\%: 41-63\%) for colon capsule endoscopy and $45 \%$ (CI 95\%: 33-57\%) for conventional colonoscopy: not indicating a significant difference. Procedural adverse events were rarely reported by patients for either investigation. The tolerability was high for both colon capsule endoscopy and conventional colonoscopy. Patient preferences for conventional colonoscopy and colon capsule endoscopy were not significantly different. Procedural adverse events were rare and the tolerability for colon capsule endoscopy was consistently reported higher or equal to that of conventional colonoscopy.
\end{abstract}

Keywords: colonoscopy; colon capsule endoscopy; patient-reported outcomes; endoscopy

\section{Introduction}

Early-stage detection and removal of colorectal pre-cancerous polyps is an effective measure for the prevention of colorectal cancer (CRC) [1]. To date, conventional optical colonoscopy (COC) is the current reference standard for examining the mucosal lining. 
However, in $5-20 \%$ of patients referred for COC, the procedure is incomplete due to insufficient bowel cleansing or patient intolerance [2-4]. Capsule endoscopy (CE) technology was initially developed to study the small bowel (SB) mucosa [5]. The SB capsule proved to be exceptionally tolerated by patients, which boosted further research in developing capsules for other parts of the gastrointestinal (GI) tract. In 2006, the first generation of the colon capsule endoscope (CCE1), equipped with a twin set of cameras, was introduced as a promising diagnostic alternative to COC [6]. Due to mediocre results in large trials, the CCE1 was replaced by the improved second-generation CCE (CCE2), currently in use, with reported adenoma detection rates equal to $\mathrm{COC}[7,8]$. However, the lack of steerability and camera focus control does no extra favors to CCE. In the wake of the COVID-19 pandemic, the technology is being increasingly adopted in clinical practice in England and Scotland to ease overburdened healthcare systems $[9,10]$. Conversely, CCE may be performed as an out-patient procedure with minimal involvement of healthcare professionals; moreover, the incidence of severe adverse events (AE) is very low. Patients undergoing CCE have been reported with less discomfort and embarrassment in many studies, juxtaposed against COC [11].

The existing knowledge on patient discomfort and minor AE with CCE is scarce as patients only have renewed contact with the hospital if a subsequent COC is mandatory and, even so, patient-reported outcomes (PRO) are rarely addressed. Patient acceptance and preference towards CCE are of outermost importance for a broader clinical implementation. Hence, we find it relevant to conduct a systematic review and meta-analysis to collect and present existing data on PRO and the preference for CCE compared to COC.

\section{Materials and Methods}

\subsection{Data Sources and Search Strategy}

We conducted a systematic literature search in PubMed, Embase and Web of Science to identify all relevant citations for studies in which acceptability, tolerance or preference was expressed by the patients (i.e., PRO), after COC and CCE were performed. The primary outcome of our analyses were the preference proportions, whereas acceptability and tolerance were secondary outcomes. According to the PICO search strategy, search terms were included in three areas: investigation, comparator and outcome. The terms within each area were combined using the Boolean "OR"; the three areas were combined in strings using the Boolean "AND". Free text search terms with truncation were included. The literature search was concluded on 12 January 2021. The complete search strings are available in Appendix A. The study was registered at the PROSPERO international register of systematic reviews (ID: CRD42021231718).

\subsection{Inclusion and Exclusion Criteria}

Inclusion criteria were:

- full text articles;

- $\quad$ articles reporting PRO after undergoing both COC and CCE or randomized controlled trials (RCT);

- articles in English/Danish/Italian/French/Spanish language.

Exclusion criteria were:

- non-RCT articles in which patients underwent either COC or CCE alone;

- the following article types: reviews, conference papers, case reports.

\subsection{Screening of References}

After identification and exclusion of duplicates, references were independently screened by three authors (U.D., P.C.V., T.B.-M.). Each author screened two thirds of the references in title and abstract, excluding those not meeting the inclusion criteria. In case of discrepancy, the reference was included for full text evaluation. After this first 
step, the process was repeated on included references by evaluating full texts with the same modality.

\subsection{Data Extraction}

All data were extracted in accordance with the Preferred Reporting Items for Systematic Reviews and Meta-Analyses (PRISMA) [12]. We collected demographic data of the patients, the setting for intervention, the type of CE (CCE1, CCE2 or PillCam ${ }^{\circledR}$ Crohn), cleanliness and completion rates for both COC and CCE. COC procedures were considered complete when the caecum was reached; $\mathrm{CE}$ procedures were considered complete when the $\mathrm{CE}$ was excreted during the recording time or when the anal verge or the hemorrhoidal plexus were visualized. PRO and AE were also collected. Regarding PRO, the preference for COC and CCE was expressed as proportions (\%). AE were limited to events related to the procedure itself, excluding those related to bowel preparation (BP).

\subsection{Study Assessment and Risk of Bias}

A study assessment of the included studies was performed by three independent reviewers (UD, PCV, TBM) through the STROBE assessment tool and the Cochrane Collaboration's tool (according to the study design) [13,14]. STROBE items $6 \mathrm{~b}, 12 \mathrm{~d}, 14 \mathrm{c}$ and $16 \mathrm{c}$ were omitted as they were not applicable to the included studies.

For STROBE, we arbitrarily designated studies as of low-, medium- and high transparency and completeness according to the tools' outcome scores: for STROBE, the cut-offs were respectively $60 \%$ and $80 \%$ of the total maximum points $(n=30)$. For Cochrane, within each bias category (selection, performance, detection, attrition, reporting), the bias level was rated as low, high or unclear.

Although aware that the primary aim of STROBE and Cochrane tools is to improve the overall quality of reporting, we empirically assumed that the higher number of points in STROBE and higher number of low risk of bias assessments in Cochrane, the higher the transparency and completeness of the included study.

\subsection{Statistical Analysis}

To evaluate the proportion of patients preferring CCE or COC, respectively, we calculated the preference proportions of CCE and COC. This was defined as the proportion of patients preferring each out of the total number of patients responding. Patients responding to data collection (interview or questionnaire) but not indicating preference were included in the denominator. Non-responders were excluded. The significance level was set at $5 \%$, and $95 \%$ confidence intervals (CI) were calculated. All pooled estimates were calculated in random effects models using the Freeman-Tukey double arcsine transformation. In sensitivity analyses, we repeated the preference proportions calculations, first, after exclusion of studies from which only a subset of the sample could be included, and second, after exclusion of studies with samples under 50 individuals. $\mathrm{I}^{2}$ statistics were performed to test and evaluate the heterogeneity by applying thresholds provided by the Cochrane Handbook [15]. Potential sources of heterogeneity were tested by univariate meta-regressions. To investigate publication bias and small study effects, Egger's tests [16] were performed and illustrated by funnel plot. Individual study data were extracted and compiled in spreadsheets for pooled analyses. Data analyses were conducted using Stata 16 (StataCorp, College Station, TX, USA, 2019. Stata Statistical Software: Release 16, StataCorp LLC) including the metaprop command [17].

\section{Results}

\subsection{Search}

The initial literature search resulted in 1632 references. After the removal of duplicates this was reduced to 1326 and, additionally, 1274 were excluded after title and abstract screening. Full text screening was performed for 52 references and 14 were eventually included in the study (Figure 1) [11,18-30]. In 12 studies, participants had undergone 
both CCE and COC, and in two $(n=2)$ studies they were randomized for either. Included studies are described in Table 1 . Ten $(n=10)$ studies reported patient preference for CCE and eight $(n=8)$ reported patient preference for COC. Four studies did not report patient preference but described patient-reported $\mathrm{AE}$ and/or tolerability.

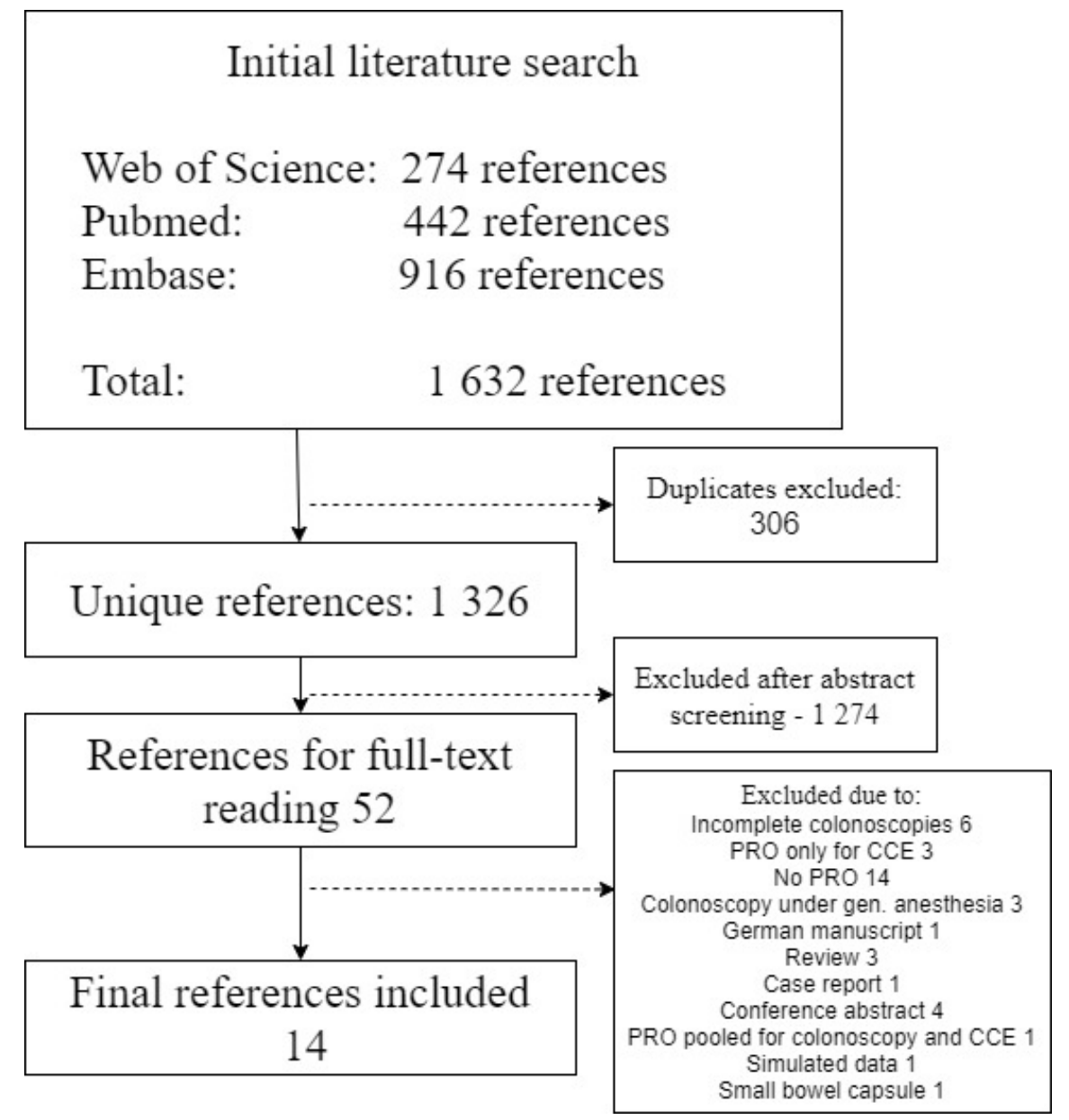

Figure 1. Consort diagram of the systematic review. Abbreviations: CCE, colon capsule endoscopy; PRO, patient-reported outcomes.

Table 1. Study characteristics.

\begin{tabular}{|c|c|c|c|c|c|c|}
\hline Author-Year & Country & Study Type & $\begin{array}{c}\text { PR CCE } \\
\text { Preference }\end{array}$ & $\begin{array}{l}\text { PR COC } \\
\text { Preference }\end{array}$ & PR AE & $\begin{array}{c}\text { PR } \\
\text { Tolerability }\end{array}$ \\
\hline Adrian-De-Ganzo 2015 [18] & Spain & RCT & Yes & Yes & Yes & No \\
\hline Alvarez-Urturi 2017 [19] & Spain & OBS & Yes & Yes & Yes & Yes \\
\hline Brechmann 2016 [20] & Germany & OBS & Yes & Yes & Yes & No \\
\hline Bruining 2020 [21] & USA, Austria, Israel & OBS & Yes & Yes & No & No \\
\hline Cash 2020 [22] & USA & $\mathrm{RCT}$ & Yes & Yes & Yes & No \\
\hline D'Haens 2015 [23] & Netherlands, Belgium & OBS & Yes & No & Yes & Yes \\
\hline Eliakim 2010 [24] & Israel & OBS & No & No & Yes & No \\
\hline Fiorillo 2020 [25] & France & OBS & No & No & Yes & Yes \\
\hline Groth 2012 [26] & Germany & OBS & Yes & Yes & Yes & No \\
\hline Meister 2013 [27] & Germany & OBS & No & No & Yes & Yes \\
\hline Pilz 2010 [28] & Switzerland & OBS & Yes & Yes & Yes & No \\
\hline Shi 2017 [29] & Hong Kong & OBS & Yes & No & Yes & Yes \\
\hline Thygesen 2019 [11] & Denmark & OBS & No & No & Yes & Yes \\
\hline Voska 2019 [30] & Czech Republic & OBS & Yes & Yes & Yes & No \\
\hline
\end{tabular}

Abbreviations: AE, adverse events; CCE, colon capsule endoscopy; COC, conventional optical colonoscopy; OBS, observational; PR, patient-reported; RCT, randomized controlled trial. 
Nine $(n=9)$ studies were evaluated as having high transparency and completeness and five $(n=5)$ studies as medium, see Appendix B.

\subsection{Patient Preference}

Pooled preference estimates were 52\% (95\% CI 41;63) (Figure 2) for CCE and $45 \%$ (95\% CI 33;57) (Figure 3) for COC, not indicating a significant difference. Preference for CCE varied between studies from $13 \%$ to $82 \%$, whereas the range for COC preference was $18 \%$ to $69 \%$. Reported reasons for preferring CCE included less invasiveness, no need for sedation or driver, only one investigation for all GI segments, no need for intravenous access, less embarrassment and discomfort, the mobility and access to investigation and, finally, fear of COC-related discomfort or complications. Reported reasons for preferring COC included it being a familiar procedure, the opportunity to perform biopsies, less time-consuming, less BP, it being a standard procedure and that CCE restricts daily life activities. The sensitivity analysis excluding studies with only a subset population showed similar estimates where the pooled preference estimates were $54 \%$ (95\% CI $42 ; 65)$ for CCE and $45 \%(95 \%$ CI $32 ; 58)$ for COC, not indicating a significant difference. The sensitivity analysis excluding small size studies showed similar estimates where the pooled preference estimates were $48 \%(95 \%$ CI $35 ; 61)$ for CCE and $49 \%(95 \%$ CI $35 ; 64)$ for COC, not indicating a significant difference.

As substantial heterogeneity was present in both preference proportion estimations $\left(\mathrm{I}^{2}=88.32 \%\right.$ and 85.81$)$, univariate meta-regressions were performed to identify possible sources. None of the tested variables resulted in statistically significant effects, although age mean yielded the lowest observed $p$-values for both CCE and COC preference analyses (Table 2). Funnel plots are included (Figure 4) illustrating possible publication bias. Egger's tests for small study effects were performed for each pooled preference proportion estimation with $p$-values of 0.084 for CCE and 0.040 for COC, indicating the presence of small study effects, at least for the COC estimate.

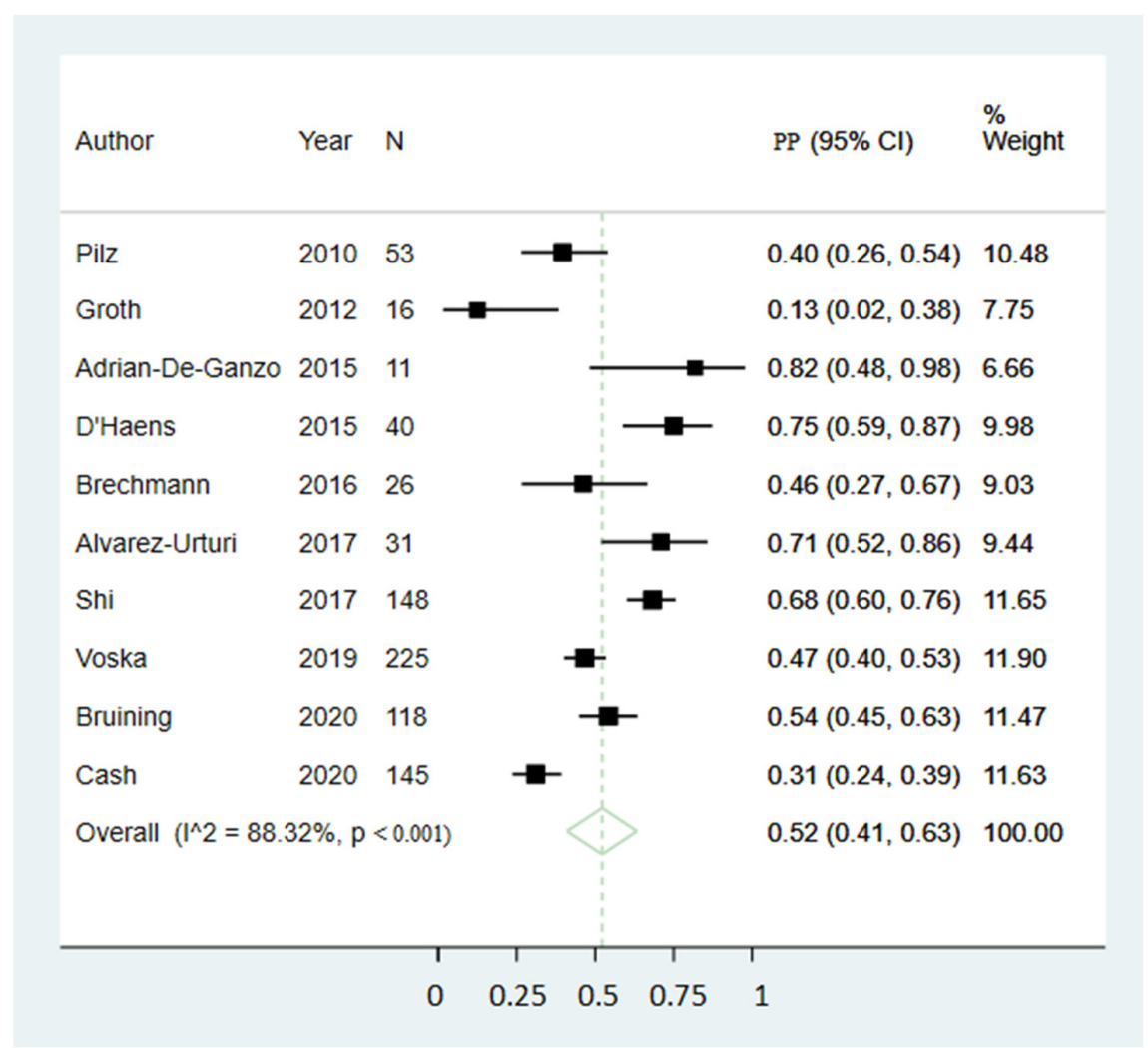

Figure 2. Pooled estimate of CCE preference proportion. Abbreviations: CI, confidence interval; PP, Estimated preference proportion. 


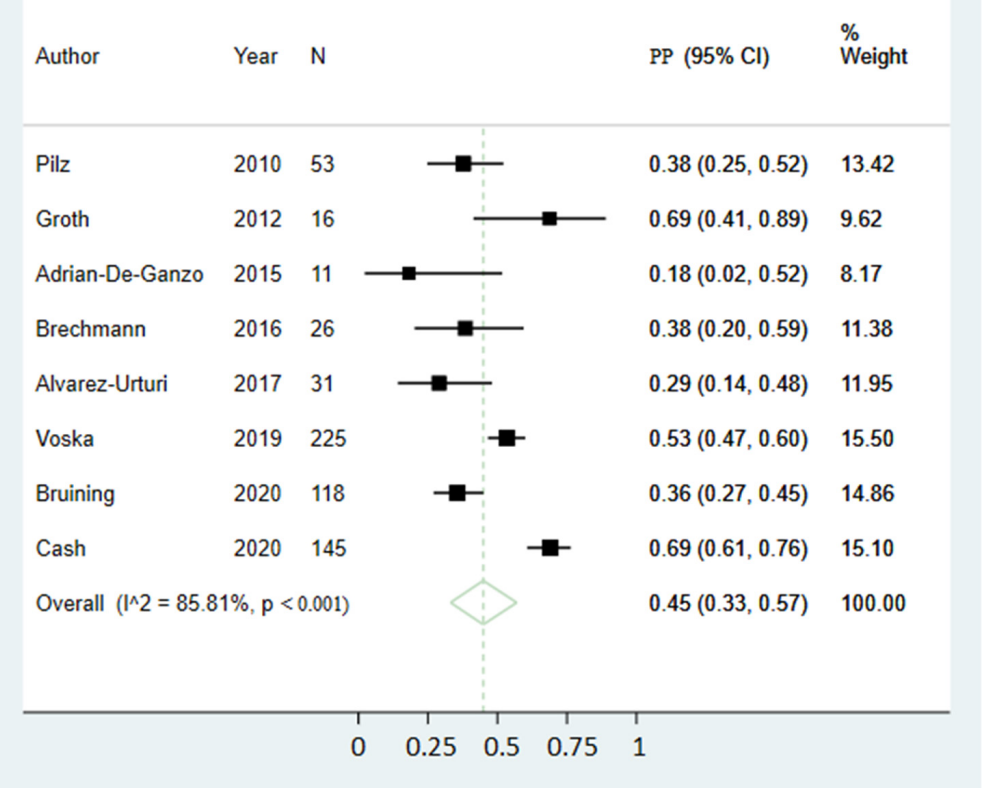

Figure 3. Pooled estimate of colonoscopy preference proportion. Abbreviations: CI, confidence interval; PP, Estimated preference proportion.
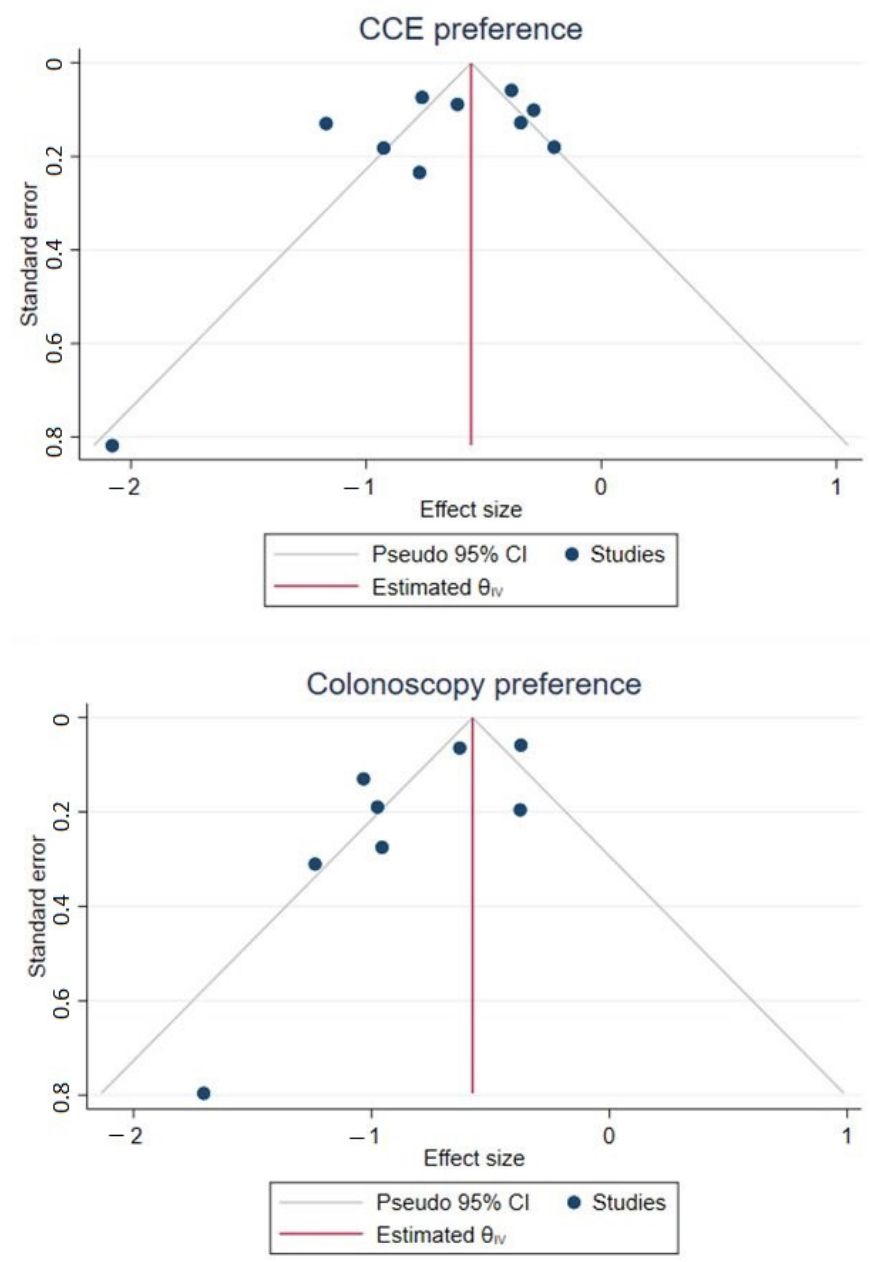

Figure 4. Funnel plots. 
Table 2. Univariate meta-regressions.

\begin{tabular}{|c|c|c|c|c|}
\hline Colon Capsule Endoscopy & & & & \\
\hline Variable & Coefficient & Lower CI 95\% & Upper CI 95\% & $p$-value \\
\hline Year published (continuous) & -0.003 & -0.081 & 0.074 & 0.936 \\
\hline Year published (categorical, before vs. after 2016) & -0.058 & -0.517 & 0.401 & 0.804 \\
\hline Sample size (continuous) & -0.002 & -0.004 & 0.001 & 0.319 \\
\hline Study type (RCT vs. OBS) & 0.116 & -0.433 & 0.665 & 0.679 \\
\hline Centers (multi vs. single) & -0.078 & -0.554 & 0.397 & 0.746 \\
\hline Modality of data collection (oral vs. questionnaire) & -0.209 & -0.660 & 0.241 & 0.362 \\
\hline Age mean (continuous) & -0.023 & -0.046 & $<0.001$ & 0.054 \\
\hline Male percentage (continuous) & -0.013 & -0.042 & 0.015 & 0.366 \\
\hline Completion rate (continuous) & -0.003 & -0.024 & 0.018 & 0.772 \\
\hline Completion rate (categorical) & -0.018 & -0.481 & 0.444 & 0.938 \\
\hline Capsule type (CCE2 vs. CCE1) & 0.190 & -0.363 & 0.742 & 0.501 \\
\hline Capsule type (PillCam Crohn ${ }^{\circledR}$ vs. CCE1) & 0.142 & -0.709 & 0.994 & 0.743 \\
\hline Patient group (symptomatic vs. screening) & -0.045 & -0.856 & 0.766 & 0.913 \\
\hline Patient group (chronic illness vs. screening) & 0.301 & -0.163 & 0.764 & 0.204 \\
\hline Evaluation of study (STROBE/Cochrane) & 0.040 & -0.520 & 0.601 & 0.887 \\
\hline \multicolumn{5}{|l|}{ Colonoscopy } \\
\hline Variable & Coefficient & Lower CI 95\% & Upper CI 95\% & $p$-value \\
\hline Year published (continuous) & 0.010 & -0.063 & 0.083 & 0.797 \\
\hline Year published (categorical, before vs. after 2016) & 0.063 & -0.487 & 0.612 & 0.823 \\
\hline Sample size (continuous) & 0.002 & -0.002 & 0.005 & 0.370 \\
\hline Study type (RCT vs. OBS) & -0.345 & -0.879 & 0.189 & 0.206 \\
\hline Centers (multi vs. single) & -0.017 & -0.609 & 0.575 & 0.955 \\
\hline Modality of data collection (oral vs. questionnaire) & -0.024 & -0.613 & 0.565 & 0.937 \\
\hline Age mean (continuous) & 0.022 & -0.009 & 0.053 & 0.155 \\
\hline Male percentage (continuous) & 0.004 & -0.024 & 0.032 & 0.772 \\
\hline Completion rate (continuous) & 0.057 & -0.035 & 0.150 & 0.223 \\
\hline Completion rate (categorical) & 0.006 & -0.632 & 0.644 & 0.985 \\
\hline Patient group (symptomatic vs. screening) & -0.267 & -1.103 & 0.568 & 0.531 \\
\hline Patient group (chronic illness vs. screening) & -0.345 & -1.033 & 0.343 & 0.326 \\
\hline Evaluation of studies (STROBE/Cochrane) & 0.036 & -0.533 & 0.606 & 0.900 \\
\hline
\end{tabular}

Abbreviations: CCE, colon capsule endoscopy; $\mathrm{CI}$, confidence interval; OBS, observational; RCT, randomized controlled trial.

\subsection{Adverse Events}

Very low proportions of AE (often none) were reported in the included studies. Only one $(n=1)$ moderate/severe $\mathrm{AE}$ (i.e., capsule retention) was reported in CCE from patients. More retentions could be expected as several of the included studies report retrieving capsules in the following colonoscopy if not excreted. Mild patient-reported AE from CCE included difficulty with camera ingestion and abdominal discomfort. In comparison, two $(n=2)$ moderate/severe $\mathrm{AE}$ in COC were reported from patients (i.e., colonic perforation). Mild patient-reported AE from COC included local phlebitis due to intravenous access, abdominal pain, rectal bleeding and elevated blood pressure. In conclusion, moderate to severe procedural AE were rarely reported by patients for both CCE and COC.

\subsection{Tolerability}

Several studies reported results that were collected using visual analog scales (VAS) and/or numerical scales. Median overall rating reported from 1 (very bad) to 10 (very good) was 8 for CCE and 7 for COC in 148 individuals [29]. On a numerical scale ranging from 1 to 10, 238 patients reported their discomfort level from COC and 239 from CCE. Low level discomfort $(<4)$ was reported by $35.2 \%$ for colonoscopy versus $88.5 \%$ for CCE. High level discomfort was reported by $27.2 \%$ for COC versus $0.4 \%$ for CCE [11]. Using a five-point scale rating from none through mild, moderate, severe and intolerable, the embarrassment was rated severe or worse in 39\% for COC and $8 \%$ for CCE by 40 patients. 
For discomfort this was $42 \%$ for COC and $11 \%$ for CCE [23]. Thirteen $(n=13)$ ulcerative colitis patients rated overall tolerability for CCE and COC on a ten-point scale to be 7.9 average for both procedures, even though they rated abdominal pain 4.9 for COC and 1.0 for CCE on the same scale [27]. Similar results were reported from 32 patients with previous colorectal surgery who reported a mean tolerance score of 8.50 for CCE and 8.56 for COC on a ten-point VAS. The same group rated comfort of the procedure to be 8.56 for CCE and 8.63 for COC [24]. In 31 individuals with familial CRC, everyone rated CCE to be good or better, compared to $84 \%$ for colonoscopy, on a five-point scale (poor, fair, good, very good and excellent) [19].

\section{Discussion}

Our systematic review with meta-analysis shows no significant difference in patients' preference between COC and CCE. About half of the included patients, having tried both modalities, declare an equal preference for the two colonic imaging modalities. One would have expected that patient preference would sway towards CCE as it can provide a virtually painless exploration of the colon, with similar diagnostic yield and accuracy to COC in many clinical situations. Nevertheless, all that glitters is not gold: as highlighted in the results of our study, the watershed of PRO for CCE versus COC remains blurred; the stigma surrounding colonoscopy, often raising well-known barriers in the CRC screening populations, failed to show any apparent inclination towards CCE as one may have expected, concerning PRO. On the other hand, a substantial proportion of patients still preferred CCE even though COC is considered the golden standard. The reality may be that each investigation should target the patient in question and not be considered a one-test-fits-all strategy.

In a recent interim analysis [31], we asked a screening population, before receiving their result from a submitted fecal immunochemical test, to indicate which investigation they would prefer. In 14,461 individuals, 50.0\% chose CCE and 9.9\% preferred COC, while those remaining did not mark a preference. The preference for CCE before the investigation compared to after seems similar (i.e., as in the present study), while the preference for COC is much higher after the procedure, indicating that the expectations for colonoscopy may be much worse than the actual experience.

In the current study, no statistically significant possible sources of heterogeneity were highlighted regarding patients' preference. The age of the patients in both CCE and COC groups was the variable with the lowest $p$-value. As speculation, a slight tilt in preference towards COC in older patients may be explained by the higher pre-test probability of colorectal pathology, eventually leading to therapeutic COC after CCE anyway. Taking into consideration possible publication bias, the funnel plot for COC preference seems a bit skewed to the left (although mostly clustered with few outliers), whereas for CCE it is more homogenous.

Taking into account these data, when coming to tolerability parameters, surprisingly, the tolerability was consistently reported higher for CCE. It is unclear why this does not translate to higher patient preference for the modality. One explanation may be that the patient-reported advantages for COC outweigh the drawbacks and these are therefore accepted. Moreover, patients with known disease who are familiar with COC, may be more accepting of the procedure and any level of relevant discomfort, or since the tolerability is very high for both procedures, the relative higher tolerance for CCE has no actual behavioral relevance. Moreover, if the tolerance is very high for both, the risk of two examinations following a positive CCE could be considered too much of a risk.

Both examinations also require two stints of BP as the CCE report is not available immediately after excretion, enabling COC under same preparation. As BP is a documented barrier to $\mathrm{COC}$ and the increased cleansing regimen for $\mathrm{CCE}$ is a reason for preferring COC [22,32], the risk of double BP may tilt the preference towards COC, thereby playing an important role in the expression of PRO. As patient-reported procedural AE were very limited, they probably did not affect preference to a high degree, although the type of severe 
AE reported from COC (perforation) compared to CCE (capsule retention) is probably more likely to cause long-term effects. However, to the contrary, perforation is more common in therapeutic colonoscopies, and CCE is not suited for individuals in need of therapeutic $\operatorname{COC}[33,34]$.

This study comes with some limitations. The presence of small study effects for pooled preference proportion for COC (as backed up by the Egger's tests) introduces the risk of publication bias. The $\mathrm{I}^{2}$ statistics indicated heterogeneous subsets of patients, and different collection methods were used in the assessments of PRO (oral interview in person, by phone or questionnaire), although no statistically significant sources were identified. Finally, for some of the included studies, only a subset of patients could be included in the meta-analysis in this review as only those subsets underwent both investigations. This introduces a risk of overrepresentation of patients with positive CCE as those excluded in the present study were discharged after negative CCE. It is expected that COC is preferred in patients with pathology as they would prefer going straight to COC since it is not possible to perform a biopsy or polypectomy with CCE.

\section{Conclusions}

The preference proportions for CCE and COC were not statistically different in this meta-analysis, with an estimated $52 \%$ preferring CCE and $45 \%$ preferring COC. Procedural $\mathrm{AE}$ are rare in both CCE and COC. The tolerability for CCE is generally reported higher or equal to that of COC. As a reminder for future studies, it should be emphasized to include both patients with and without positive CCE/COC to be able to estimate preference proportions for the general population.

Author Contributions: Conceptualization, T.B.-M.; methodology, U.D., P.C.V. and T.B.-M.; software, U.D.; validation, U.D., P.C.V. and T.B.-M.; formal analysis, U.D., P.C.V. and T.B-M.; data curation, U.D., P.C.V. and T.B.-M.; writing-original draft preparation, U.D., P.C.V. and T.B.-M.; writing-review and editing, U.D., P.C.V., A.K., G.B., E.T., C.S., I.F.-U., M.P. and T.B.-M.; visualization, U.D., P.C.V. and T.B.-M.; supervision, A.K., G.B., E.T., C.S., I.F.-U., M.P.; project administration, U.D., P.C.V. and T.B.-M. All authors have read and agreed to the published version of the manuscript.

Funding: This research received no external funding.

Institutional Review Board Statement: Not applicable.

Informed Consent Statement: Not applicable.

Data Availability Statement: All data in this study were obtained from already published material in scientific journals, referenced in the paper, and can be obtained by any individual with access to these.

Conflicts of Interest: The authors declare no conflict of interest.

\section{Appendix A}

Appendix A.1. PICO

Question: Are patient-reported outcomes for colon capsule endoscopy (CCE) superior to colonoscopy?

P: Patient subjected to either colonoscopy or/and CCE

I: Colon capsule endoscopy

C: Colonoscopy

O: All patient-reported outcomes e.g., pain, satisfaction, tolerability and days off from work. 
Table A1. Pico scheme search terms.

\begin{tabular}{|c|c|c|}
\hline Investigation & Comparator & Outcome \\
\hline $\begin{array}{c}\text { Capsule camera* } \\
\text { Wireless camera* } \\
\text { Wireless camera endoscop* } \\
\text { WCE } \\
\text { CCE } \\
\text { Colon capsule endoscop* } \\
\text { PillCam* } \\
\text { Pill Cam* } \\
\text { Camera pill* } \\
\text { Capsule endoscop* } \\
\text { Endoscop*, Capsule } \\
\text { Wireless Capsule Endoscop* } \\
\text { Capsule Endoscop*, Wireless } \\
\text { Endoscop*, Wireless Capsule } \\
\text { Video Capsule Endoscop* } \\
\text { Capsule Endoscop*, Video } \\
\text { Capsule Endoscopy, Video } \\
\text { Endoscop*, Video Capsule } \\
\text { Pan-enteric capsule endoscop* }\end{array}$ & $\begin{array}{c}\text { Colonoscopy(MESH) } \\
\text { Colonoscop* } \\
\text { Colonoscopic Surgical Procedure* }^{*} \\
\text { Procedure*, Colonoscopic Surgical }^{*} \text { Surgical Procedure* }{ }^{*} \text { Colonoscopic } \\
\text { Surger }{ }^{*} \text { Colonoscopic } \\
\text { Surgical Procedure*, Colonoscopic } \\
\text { Colonoscopic Surger* } \\
\text { Colonoscopes(MESH) } \\
\text { Colonoscope }\end{array}$ & $\begin{array}{c}\text { Patient-related outcome } \\
\text { Patient acceptance of health care(MESH) } \\
\text { Health Care Utilization } \\
\text { Utilization, Health Care } \\
\text { Patient Acceptance of Healthcare } \\
\text { Healthcare Patient Acceptance* } \\
\text { Nonacceptor* of Health Care } \\
\text { Care Nonacceptor*, Health } \\
\text { Health Care Nonacceptor* } \\
\text { Health Care Seeking Behavior } \\
\text { Acceptor* of Health Care } \\
\text { Care Acceptor*, Health } \\
\text { Health Care Acceptor* } \\
\text { Health Care Acceptabili* } \\
\text { Acceptability of Healthcare } \\
\text { Healthcare Acceptabilit* } \\
\text { Patient satisfaction(MESH) } \\
\text { Patient preference(MESH) } \\
\text { Patient Preference*. patient } \\
\text { Patient comfort(MESH) } \\
\text { Comfort, patient } \\
\text { Comfort care } \\
\text { Care, comfort } \\
\text { Patient Confidence } \\
\text { Tolerance } \\
\text { Compliance } \\
\text { Preference } \\
\text { Patient-reported treatment outcome } \\
\text { Patient-reported } \\
\text { patientreported } \\
\text { Self-reported } \\
\text { Selfreported } \\
\text { Interview* } \\
\text { Questionnaire* } \\
\text { Adverse event* } \\
\text { Minor complication* } \\
\text { Major complication* } \\
\text { Complication* } \\
\text { Adverse effect* } \\
\text { Adverse reaction* } \\
\text { PROM* }\end{array}$ \\
\hline
\end{tabular}

${ }^{*}$ represents any group of characters including no character.

Appendix A.2. Search Strings

PubMed/WES:

((Capsule camera* OR Wireless camera* OR Wireless camera endoscop* OR WCE OR CCE OR Colon capsule endoscop* OR PillCam* OR Pill Cam* OR Camera pill* OR Capsule endoscop* OR Endoscop*, Capsule OR Wireless Capsule Endoscop* OR Capsule Endoscop*, Wireless OR Endoscop*, Wireless Capsule OR Video Capsule Endoscop* OR Capsule Endoscop*, Video OR Capsule Endoscopy, Video OR Endoscop*, Video Capsule OR Pan-enteric capsule endoscop*) AND (Colonoscopy OR Colonoscop* OR Colonoscopic Surgical Procedure* OR Procedure*, Colonoscopic Surgical OR Surgical Procedure*, Colonoscopic OR Surger*, Colonoscopic OR Surgical Procedure*, Colonoscopic OR Colonoscopic Surger* OR Colonoscopes OR Colonoscope*) AND (Patient-related outcome OR Patient acceptance of health care OR Health Care Utilization OR Utilization, Health Care OR Patient Acceptance of Healthcare OR Healthcare Patient Acceptance* OR Nonacceptor* of 
Health Care OR Care Nonacceptor*, Health OR Health Care Nonacceptor* OR Health Care Seeking Behavior OR Acceptor* of Health Care OR Care Acceptor*, Health OR Health Care Acceptor* OR Health Care Acceptabili* OR Acceptability of Healthcare OR Healthcare Acceptabilit* OR Patient satisfaction OR Patient preference OR Patient Preference* OR Patient comfort OR Comfort, patient OR Comfort care OR Care, comfort OR Patient Confidence OR Tolerance OR Compliance OR Preference OR Patient-reported treatment outcome OR Patient-reported OR patientreported OR Self-reported OR Selfreported OR Interview* OR Questionnaire* OR Adverse event* OR Minor complication* OR Major complication* OR Complication* OR Adverse effect* OR Adverse reaction* OR PROM*))

Embase:

((“Capsule camera*" or "Wireless camera*" or "Wireless camera endoscop" or "WCE" or "CCE" or "Colon capsule endoscop*" or "PillCam" or "Pill Cam" or "Camera pill*" or "Capsule endoscopy/" or "Capsule endoscope/" or "Capsule endoscop*" or "Endoscop*, Capsule" or "Wireless Capsule Endoscop*" or "Capsule Endoscop*, Wireless" or "Endoscop*, Wireless Capsule" or "Video Capsule Endoscop*" or "Capsule Endoscop*, Video" or "Capsule Endoscopy, Video" or "Endoscop*, Video Capsule" or "Pan-enteric capsule endoscop*") and ("Colonoscop*" or "Colonoscopic Surgical Procedure*" or "Procedure*, Colonoscopic Surgical" or "Surgical Procedure*, Colonoscopic" or "Surger*, Colonoscopic" or "Surgical Procedure*, Colonoscopic" or "Colonoscopic Surger" or "Colonoscope ${ }^{* \prime}$ or "Colonoscope/" or "Colonoscopy/") and ("Patient-related outcome" or "Health Care Utilization" or "Utilization, Health Care" or "Patient Acceptance of Healthcare" or "Healthcare Patient Acceptance ${ }^{*}$ " or "Nonacceptor* of Health Care" or "Care Nonacceptor*, Health" or "Health Care Nonacceptor" or "Health Care Seeking Behavior" or "Acceptor* of Health Care" or "Care Acceptor", Health" or "Health Care Acceptor" or "Health Care Acceptabili*" or "Acceptability of Healthcare" or "Healthcare Acceptabilit" or "Patient Preference*. patient" or "Comfort, patient" or "Comfort care" or "Care, comfort" or "Patient Confidence" or "Tolerance" or "Compliance" or "Preference" or "Patient-reported treatment outcome/ " or "Patient-reported Patientreported outcome/" or "Self-reported" or "Selfreported" or "Interview/" or "Questionnaire/" or "Adverse event/" or "Complication/" or "Adverse effect" or "Adverse reaction*" or "PROM*" or "Patient-reported outcome/" or "Patient attitude/" or "Health Care Utilization/" or "Patient assessment/" or "Patient satisfaction/" or "Patient preference/" or "Patient comfort/"))

\section{Appendix B}

Table A2. Study evaluation of transparency and completeness.

\begin{tabular}{ccc}
\hline Author, Year & Checklist & Evaluation \\
\hline Alvarez-Urturi, 2017 & STROBE & Medium \\
Brechmann, 2016 & STROBE & Medium \\
Bruining, 2020 & STROBE & High \\
D'Haens, 2015 & STROBE & High \\
Eliakim, 2010 & STROBE & High \\
Fiorillo, 2020 & STROBE & High \\
Groth, 2012 & STROBE & Medium \\
Meister, 2013 & STROBE & Medium \\
Pilz, 2010 & STROBE & High \\
Shi, 2017 & STROBE & High \\
Thygesen, 2019 & STROBE & High \\
Voska, 2019 & STROBE & High \\
Adrian-De-Ganzo, 2015 & RoB & High (low risk of bias) \\
Cash, 2020 & RoB & High (low risk of bias) \\
\hline
\end{tabular}




\section{References}

1. Moore, J.S.; Aulet, T.H. Colorectal Cancer Screening. Surg. Clin. N. Am. 2017, 97, 487-502. [CrossRef] [PubMed]

2. Burtin, P.; Bour, B.; Charlois, T.; Ruget, O.; Calès, P.; Dauver, A.; Boyer, J. Colonic investigations in the elderly: Colonoscopy or barium enema? Aging Clin. Exp. Res. 1995, 7, 190-194. [CrossRef] [PubMed]

3. Winawer, S.J.; Fletcher, R.H.; Miller, L.; Godlee, F.; Stolar, M.H.; Mulrow, C.D.; Woolf, S.H.; Glick, S.N.; Ganiats, T.G.; Bond, J.H.; et al. Colorectal cancer screening: Clinical guidelines and rationale. Gastroenterology 1997, 112, 594-642. [CrossRef] [PubMed]

4. Anderson, M.L.; Heigh, R.I.; McCoy, G.A.; Parent, K.; Muhm, J.R.; McKee, G.S.; Eversman, W.G.; Collins, J.M. Accuracy of assessment of the extent of examination by experienced colonoscopists. Gastrointest. Endosc. 1992, 38, 560-563. [CrossRef]

5. Moglia, A.; Menciassi, A.; Schurr, M.O.; Dario, P. Wireless capsule endoscopy: From diagnostic devices to multipurpose robotic systems. Biomed. Microdevices 2006, 9, 235-243. [CrossRef] [PubMed]

6. Eliakim, R.; Fireman, Z.; Gralnek, I.M.; Yassin, K.; Waterman, M.; Kopelman, Y.; Lachter, J.; Koslowsky, B.; Adler, S.N. Evaluation of the PillCam Colon capsule in the detection of colonic pathology: Results of the first multicenter, prospective, comparative study. Endoscopy 2006, 38, 963-970. [CrossRef]

7. $\quad$ Eliakim, R.; Yassin, K.; Niv, Y.; Metzger, Y.; Lachter, J.; Gal, E.; Sapoznikov, B.; Konikoff, F.; Leichtmann, G.; Fireman, Z.; et al. Prospective multicenter performance evaluation of the second-generation colon capsule compared with colonoscopy. Endoscopy 2009, 41, 1026-1031. [CrossRef]

8. Spada, C.; Hassan, C.; Munoz-Navas, M.; Neuhaus, H.; Deviere, J.; Fockens, P.; Coron, E.; Gay, G.; Toth, E.; Riccioni, M.E.; et al. Second-generation colon capsule endoscopy compared with colonoscopy. Gastrointest. Endosc. 2011, 74, 581-589.e1. [CrossRef] [PubMed]

9. Koulaouzidis, G.; Robertson, A.; Wenzek, H.; Koulaouzidis, A. Colon capsule endoscopy: The evidence is piling up. Gut 2021. [CrossRef]

10. Koulaouzidis, A.; Marlicz, W.; Wenzek, H.; Koulaouzidis, G.; Eliakim, R.; Toth, E. Returning to digestive endoscopy normality will be slow and must include novelty and telemedicine. Dig. Liver Dis. 2020, 52, 1099-1101. [CrossRef]

11. Thygesen, M.K.; Baatrup, G.; Petersen, C.; Qvist, N.; Krøijer, R.; Kobaek-Larsen, M. Screening individuals' experiences of colonoscopy and colon capsule endoscopy; a mixed methods study. Acta Oncol. 2019, 58, S71-S76. [CrossRef] [PubMed]

12. Liberati, A.; Altman, D.G.; Tetzlaff, J.; Mulrow, C.; Gøtzsche, P.C.; Ioannidis, J.P.A.; Clarke, M.; Devereaux, P.; Kleijnen, J.; Moher, D. The PRISMA statement for reporting systematic reviews and meta-analyses of studies that evaluate healthcare interventions: Explanation and elaboration. BMJ 2009, 339, b2700. [CrossRef] [PubMed]

13. Vandenbroucke, J.P.; von Elm, E.; Altman, D.G.; Gøtzsche, P.C.; Mulrow, C.D.; Pocock, S.J.; Poole, C.; Schlesselman, J.J.; Egger, M. Strengthening the Reporting of Observational Studies in Epidemiology (STROBE): Explanation and Elaboration. Ann. Intern. Med. 2007, 147, W-163-W-194. [CrossRef]

14. Higgins, J.P.T.; Altman, D.G.; Gøtzsche, P.C.; Jüni, P.; Moher, D.; Oxman, A.D.; Savović, J.; Schulz, K.F.; Weeks, L.; Sterne, J.; et al. The Cochrane Collaboration's tool for assessing risk of bias in randomised trials. BMJ 2011, 343, d5928. [CrossRef] [PubMed]

15. Cumpston, M.; Li, T.; Page, M.; Chandler, J.; Welch, V.A.; Higgins, J.; Thomas, J. Updated guidance for trusted systematic reviews: A new edition of the Cochrane Handbook for Systematic Reviews of Interventions. Cochrane Database Syst. Rev. 2019, 10, ED000142. [CrossRef] [PubMed]

16. Egger, M.; Smith, G.D.; Schneider, M.; Minder, C. Bias in meta-analysis detected by a simple, graphical test. BMJ 1997, 315, 629-634. [CrossRef]

17. Nyaga, V.N.; Arbyn, M.; Aerts, M. Metaprop: A Stata command to perform meta-analysis of binomial data. Arch. Public Health 2014, 72, 39. [CrossRef]

18. Adrián-De-Ganzo, Z.; Alarcón-Fernández, O.; Ramos, L.; Gimeno-Garcia, A.Z.; Alonso-Abreu, I.; Carrillo, M.; Quintero, E. Uptake of Colon Capsule Endoscopy vs. Colonoscopy for Screening Relatives of Patients With Colorectal Cancer. Clin. Gastroenterol. Hepatol. 2015, 13, 2293-2301.e1. [CrossRef]

19. Alvarez-Urturi, C.; Fernández-Esparrach, M.G.; Ibáñez, I.A.; de Miguel, C.R.; Dedeu, J.M.; Bessa, X.; Córdova, H.; Pellise, M.; Balaguer, F.; Ginès, A.; et al. Accuracy of Colon Capsule Endoscopy in Detecting Colorectal Polyps in Individuals with Familial Colorectal Cancer: Could We Avoid Colonoscopies? Gastroenterol. Res. Pract. 2017, 2017, 1507914. [CrossRef] [PubMed]

20. Brechmann, T.; Schmiegel, W.; Klute, L.; Rösch, T.; Pox, C. Feasibility of a colon capsule overnight procedure followed by colonoscopy. Z. Für Gastroenterol. 2016, 54, 146-151. [CrossRef]

21. Bruining, D.H.; Oliva, S.; Fleisher, M.R.; Fischer, M.; Fletcher, J.G. Panenteric capsule endoscopy versus ileocolonoscopy plus magnetic resonance enterography in Crohn's disease: A multicentre, prospective study. BMJ Open Gastroenterol. 2020, 7, e000365. [CrossRef]

22. Cash, B.D.; Fleisher, M.R.; Fern, S.; Rajan, E.; Haithcock, R.; Kastenberg, D.M.; Pound, D.; Papageorgiou, N.P.; Fernández-Urién, I.; Schmelkin, I.J.; et al. Multicentre, prospective, randomised study comparing the diagnostic yield of colon capsule endoscopy versus CT colonography in a screening population (the TOPAZ study). Gut 2020. [CrossRef] [PubMed]

23. D’Haens, G.; Löwenberg, M.; Samaan, M.A.; Franchimont, D.; Ponsioen, C.; Brink, G.R.V.D.; Fockens, P.; Bossuyt, P.; Amininejad, L.; Rajamannar, G.; et al. Safety and Feasibility of Using the Second-Generation Pillcam Colon Capsule to Assess Active Colonic Crohn's Disease. Clin. Gastroenterol. Hepatol. 2015, 13, 1480-1486.e3. [CrossRef] [PubMed] 
24. Eliakim, R.; Yassin, K.; Niv, Y.; Metzger, Y.; Lachter, J.; Ga, E.; Sapoznikov, B.; Konikoff, F.; Leichtmann, G.; Fireman, Z.; et al. Ergebnisse eines prospektiven Multizentervergleichs der 2. Kolonkapselgeneration mit der Koloskopie. Endosk. Heute 2010, 23, 144-149. [CrossRef]

25. Fiorillo, C.; Quero, G.; Longo, F.; Mascagni, P.; Delvaux, M.; Mutter, D. Capsule Endoscopy Versus Colonoscopy in Patients with Previous Colorectal Surgery: A Prospective Comparative Study. Gastroenterol. Res. 2020, 13, $217-224$.

26. Groth, S.; Krause, H.; Behrendt, R.; Hill, H.; Börner, M.; Bastürk, M.; Plathner, N.; Schütte, F.; Gauger, U.; Riemann, J.F.; et al. Capsule colonoscopy increases uptake of colorectal cancer screening. BMC Gastroenterol. 2012, 12, 80. [CrossRef]

27. Meister, T.; Heinzow, H.S.; Domagk, D.; Dortgolz, A.; Lenze, F.; Ross, M.; Domschke, W.; Lügering, A. Colon capsule endoscopy versus standard colonoscopy in assessing disease activity of ulcerative colitis: A prospective trial. Tech. Coloproctol. 2013, 17, 641-646. [CrossRef]

28. Pilz, J.B.; Portmann, S.; Peter, S.; Beglinger, C.; Degen, L. Colon Capsule Endoscopy compared to Conventional Colonoscopy under routine screening conditions. BMC Gastroenterol. 2010, 10, 66. [CrossRef]

29. Shi, H.Y.; Chan, F.K.; Higashimori, A.; Kyaw, M.; Ching, J.Y.; Chan, H.C.; Chan, J.C.; Chan, A.; Lam, K.L.; Tang, R.S.; et al. A prospective study on second-generation colon capsule endoscopy to detect mucosal lesions and disease activity in ulcerative colitis (with video). Gastrointest. Endosc. 2017, 86, 1139-1146.e6. [CrossRef] [PubMed]

30. Voska, M.; Grega, T.; Ngo, O.; Buckova, B.; Majek, O.; Vojtechova, G.; Tacheci, I.; Benes, M.; Bures, J.; Spicak, J.; et al. The Comparison of the Efficiency of Colon Capsule Endoscopy and Optical Colonoscopy in Patients with Positive Immunochemical Fecal Occult Blood Test: Multicenter, Prospective Study. Gastrointest. Endosc. 2019, 89 (Suppl. 6), AB399. [CrossRef]

31. Deding, U.; Bjoersum-Meyer, T.; Kaalby, L.; Kobaek-Larsen, M.; Thygesen, M.K.; Madsen, J.B.; Kroijer, R.; Baatrup, B. Colon capsule endoscopy in colorectal cancer screening: Interim analyses of randomized controlled trial care for colon 2015. Endosc. Int. Open 2021.

32. Kerrison, R.S.; Sheik-Mohamud, D.; McBride, E.; Whitaker, K.L.; Rees, C.; Duffy, S.; von Wagner, C. Patient barriers and facilitators of colonoscopy use: A rapid systematic review and thematic synthesis of the qualitative literature. Prev. Med. 2021, 145, 106413. [CrossRef] [PubMed]

33. Garg, R.; Singh, A.; Ahuja, K.R.; Mohan, B.P.; Ravi, S.J.K.; Shen, B.; Kirby, D.F.; Regueiro, M. Risks, time trends, and mortality of colonoscopy-induced perforation in hospitalized patients. J. Gastroenterol. Hepatol. 2020, 35, 1381-1386. [CrossRef] [PubMed]

34. Panteris, V.; Haringsma, J.; Kuipers, E. Colonoscopy perforation rate, mechanisms and outcome: From diagnostic to therapeutic colonoscopy. Endoscopy 2009, 41, 941-951. [CrossRef] [PubMed] 